\title{
Research Experience for Community College Students in Colorado: Insights on Improving the the Pipeline of 2YC Students into the Geosciences
}

Lesley Smith, Anne Gold, Suzanne Anderson, Jennifer Taylor, Rebecca Batchelor
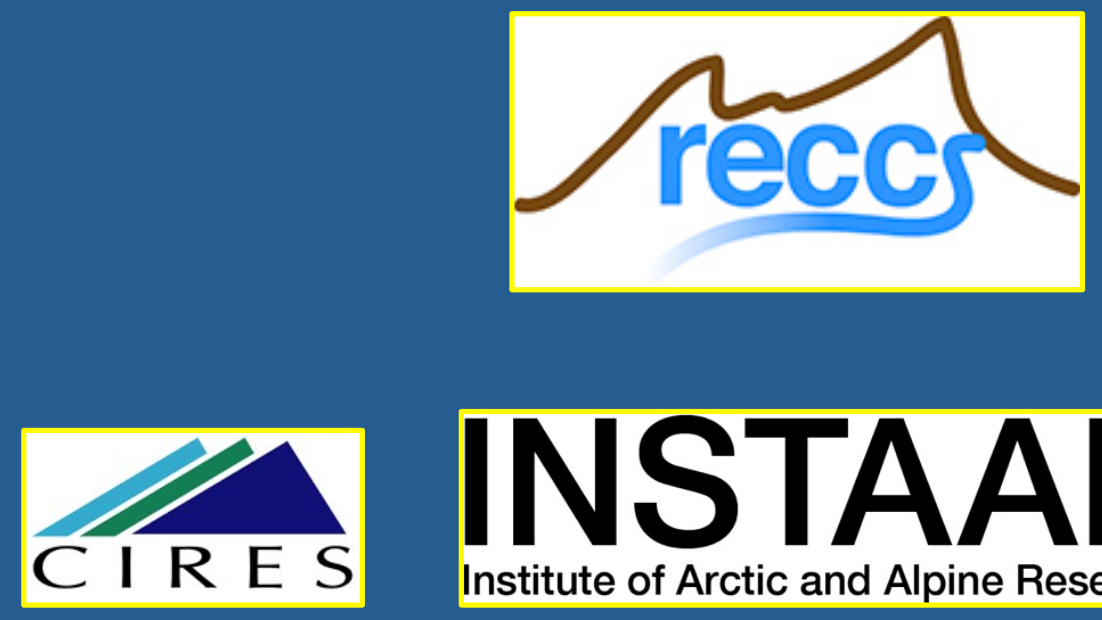


\section{Overarching Goal}

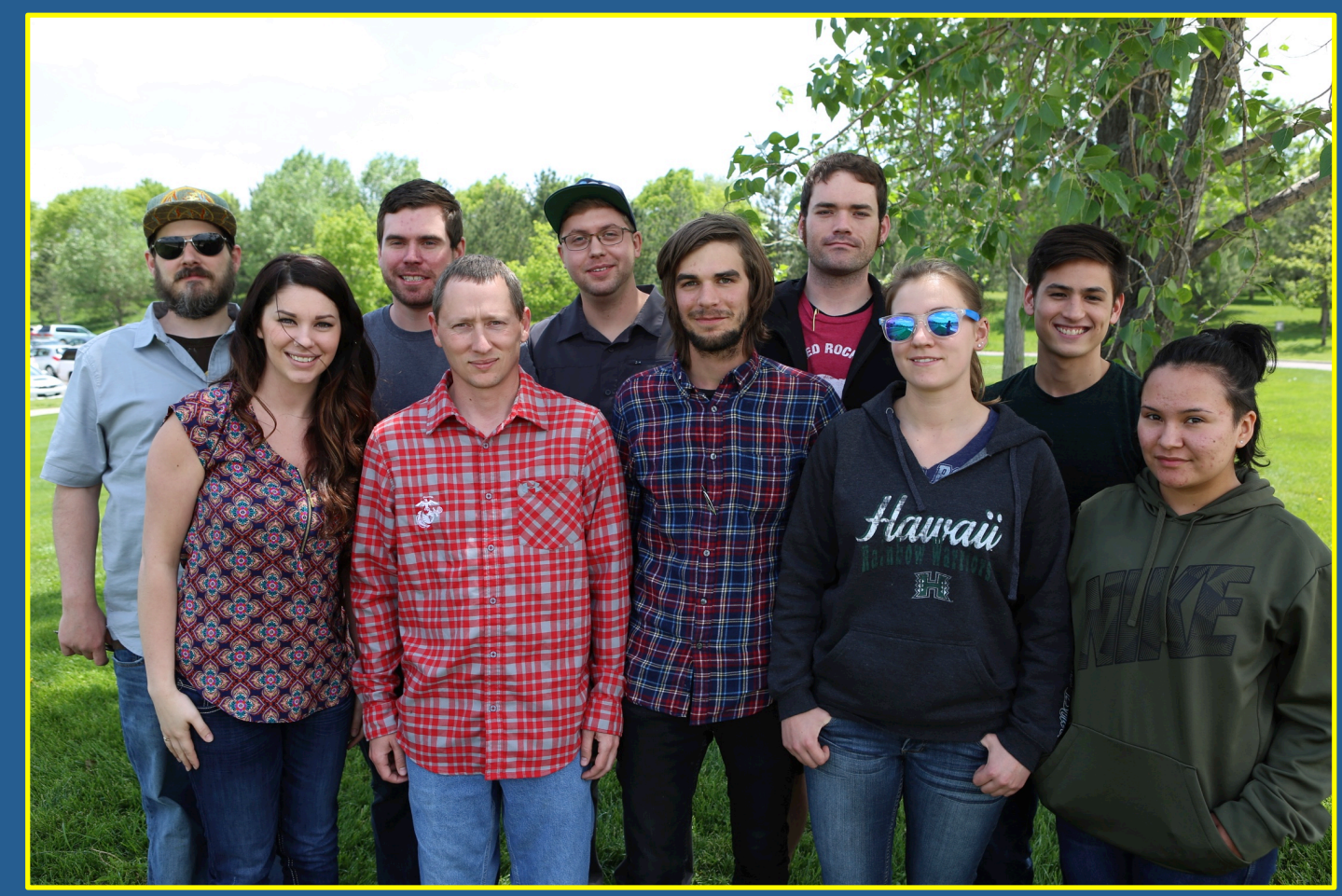

Give community college students an authentic research experience that allows them to explore geosciences and gain the confidence to successfully transition to a 4-year program in the STEM disciplines. 


\section{The Need}

\section{$100,000+$ students at 13 CO community colleges}

"Your internship program offers invaluable opportunities to our students, and are especially important to community colleges, as we typically don't have our own research projects in which to engage students. At the same time, we have many students who have an aptitude and interest in science and who can benefit greatly from hands on scientific work." President, Front Range CC

"Before the program I wasn't sure about attending a 4-year college. After speaking with the people in the program, my fellow interns, who had the same worries I did, and the people who have gone through the 4-year process and the grad student process, I am confident and I want to do it." RECCS alumnus 


\section{Evaluation}

Pre-Program:

- Individual Interview

\begin{tabular}{|c|c|}
\hline $\begin{array}{l}\text { Intro } \\
\text { Week }\end{array}$ & $\begin{array}{l}\text { First Week: } \\
\text { - } \quad \text { Perceived confidence \& Grit measures }\end{array}$ \\
\hline Week 2 & - Nraphing and ScIentinc SKIII test \\
\hline Week 3 & - Test of Science Literacy \\
\hline Week 4 & \\
\hline Week 5 & - Weekly reflection survey \\
\hline Week 6 & - Blogging \\
\hline Week 7 & End of Program: \\
\hline Week 8 & - Posttests [see first week] \\
\hline Week 9 & $\begin{array}{ll}\text { - } & \text { URSSA } \\
\text { - } & \text { Reflection Survey } \\
\text { - } & \text { Individual Interview }\end{array}$ \\
\hline
\end{tabular}

Mentor Feedback

- Check-ins

- Reflection on student 


\section{Shout-out to REU-GEO 2016!}

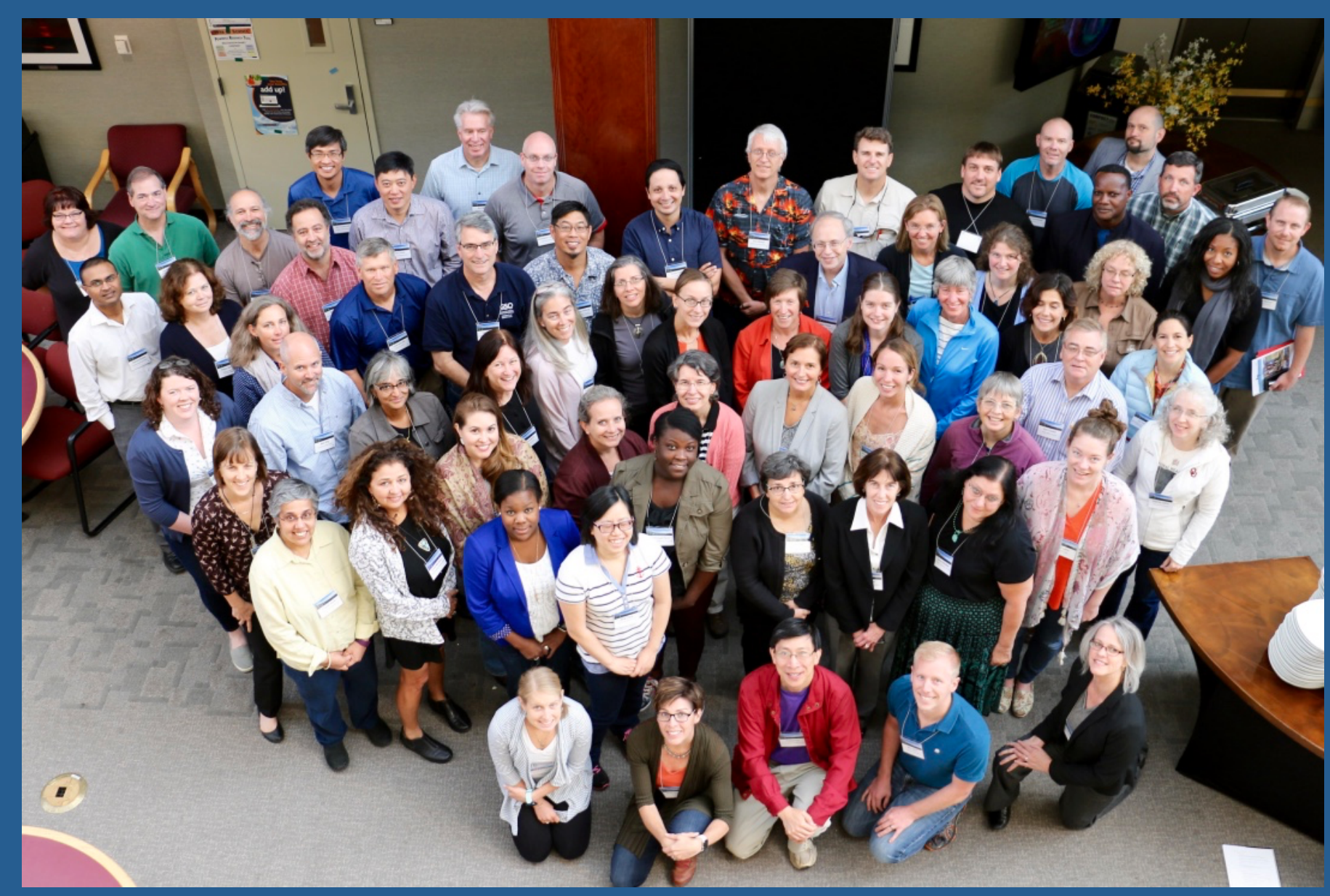




\section{Structure}

- 10 community college students for 9 weeks

- Semi-residential program

- Stipend \$500/week \& gas stipend

- Intensive first week

- 1 day/week half-day training: Communicating Science (UCAR), Excel, soft skills, ethics

- Brown bag with professionals

- Products: poster session at UCAR; oral presentation at CIRES
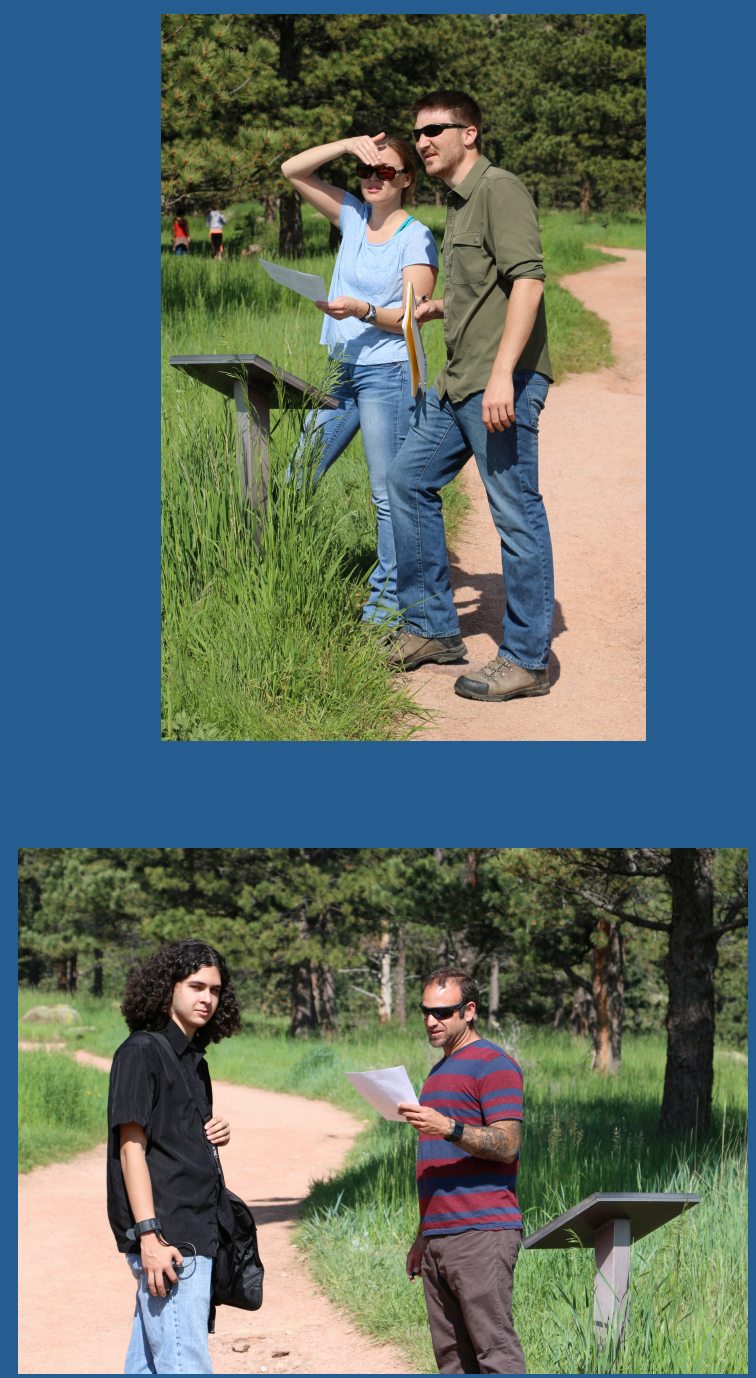


\section{First Week}

Goals:

- Set expectations

- Mentor-mentee relationship

- Build a cohort

- Begin learning to think like a scientist
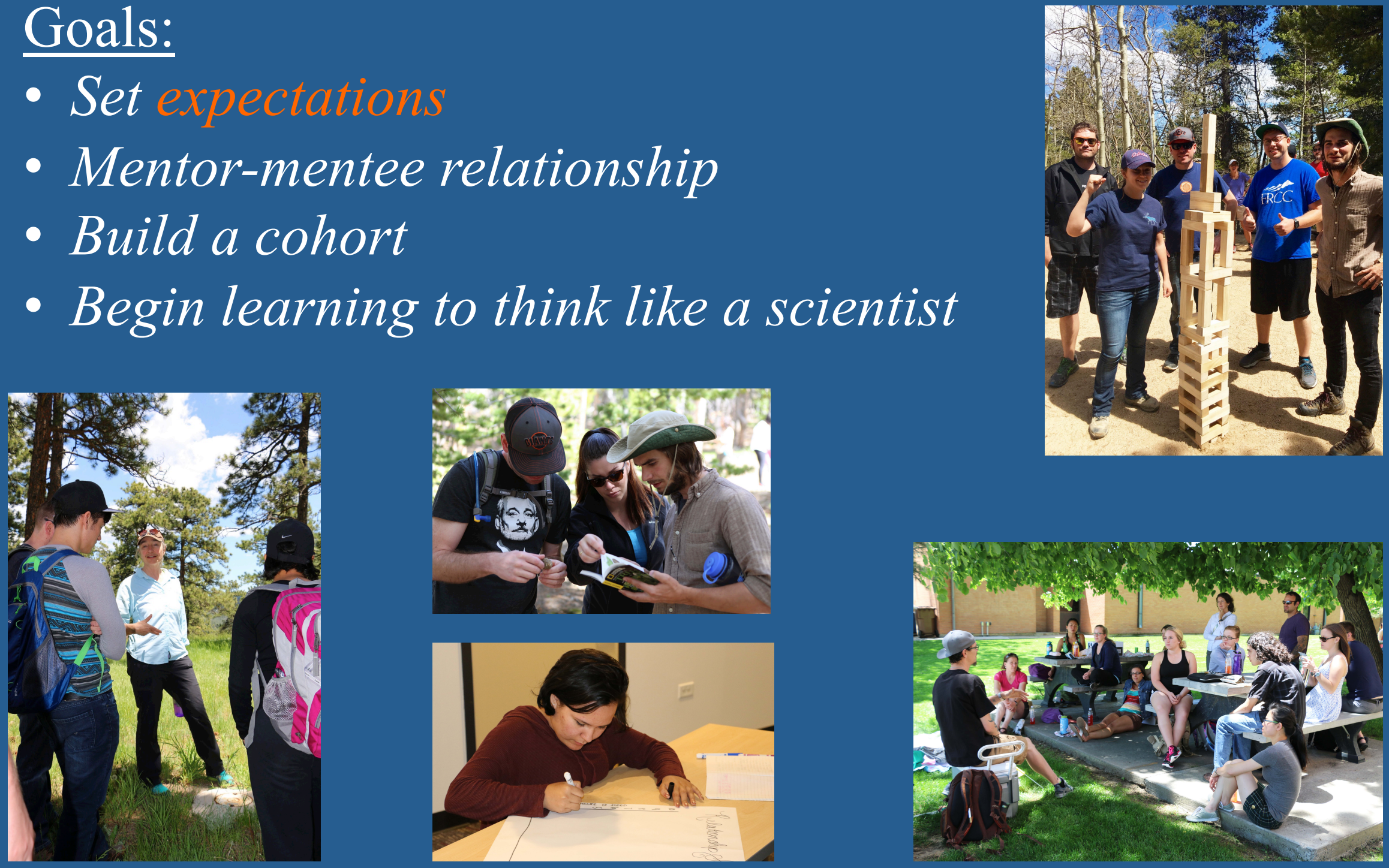


\section{Recruiting Students}

- Pilot 2014 - shotgun approach

- 2 super cheerleaders - CCD and FRCC

- CCCS meeting \& statewide networks

- Alumnae - classroom visits, posters, flyers
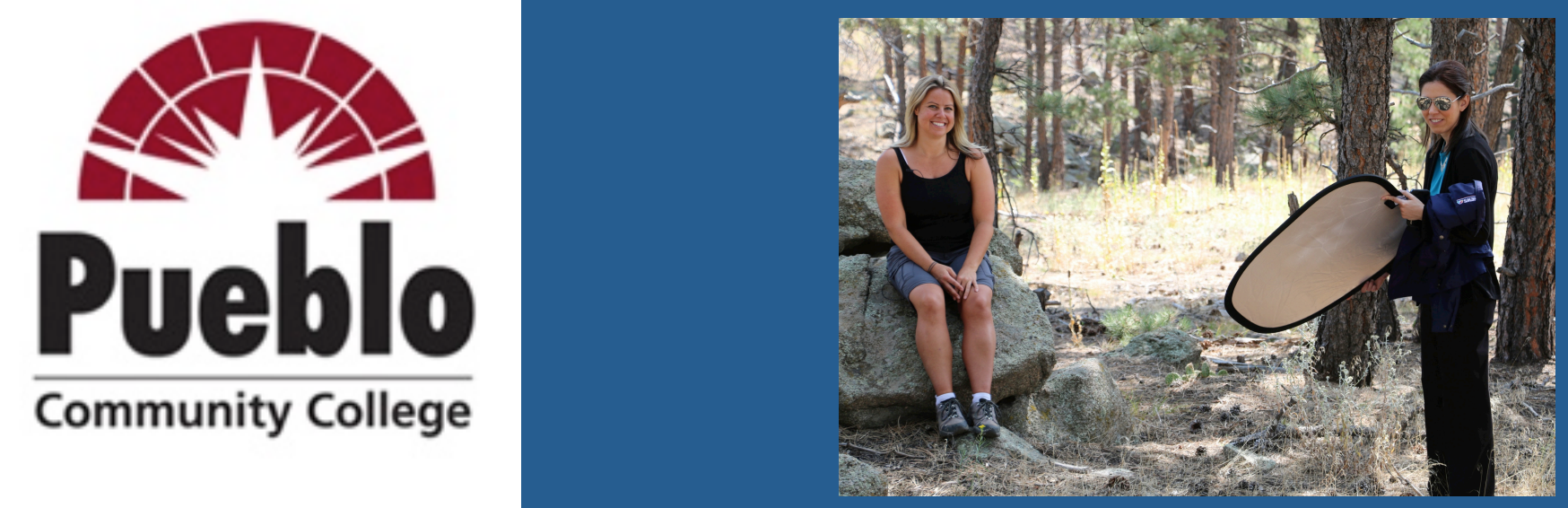


\section{Recruiting Students}

- Different educational backgrounds

- Minimum gpa; math \& science classes

- Couple of weak students each year

\section{RECCS Applications from Community Colleges}

\begin{tabular}{|l|c|c|}
\hline \multicolumn{1}{|c|}{ Community College } & $\begin{array}{c}\text { Total } \\
\text { Applicants }\end{array}$ & $\begin{array}{c}\text { Selected } \\
\text { Interns }\end{array}$ \\
\hline Aims Community College & 2 & 0 \\
\hline Arapaho Community College & 2 & 1 \\
\hline Colorado Mountain College & 2 & 0 \\
\hline $\begin{array}{l}\text { Colorado Northwestern Community } \\
\text { College }\end{array}$ & 6 & 2 \\
\hline Community College of Aurora & 4 & 1 \\
\hline Community College of Denver & 4 & 2 \\
\hline Front Range Community College & 12 & 1 \\
\hline Northeastern Junior College & - & - \\
\hline Otero Junior College & - & - \\
\hline Red Rocks Community College & 5 & 3 \\
\hline
\end{tabular}

\begin{tabular}{|l|c|c|}
\hline \multicolumn{3}{|c|}{ RECCS Applicant Demographics } \\
\hline \multicolumn{1}{|c|}{ Year } & 2015 & 2016 \\
\hline Total Applicants & 39 & 37 \\
\hline First Generation College & 10 & 13 \\
\hline Of Color & 6 & 6 \\
\hline Veteran & 1 & 1 \\
\hline
\end{tabular}




\section{Recruiting and Mentor Preparation}

Recruiting

- Robust E/O Program with longstanding relationships

- Critical Zone Observatory

- Benefits of mentoring

Preparation

- Individual meetings with mentoring group

- Weekly newsletter

- Visit lab once \& mid-program survey

Matching is tricky

No hard \& fast rules

New in 2017 - Near Peer Mentors

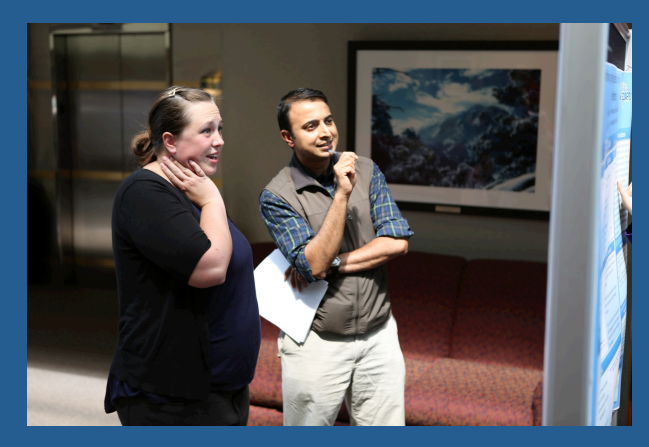




\section{Evaluation Results}

- All students successfully completed program.

- Students formed strong, supportive cohort.

- Self report: full immersion in scientific process, viewed themselves as scientists.

- Students highly motivated for STEM career at end program, including graduate school.

- Above average gains in cognitive skills and affective learning (Thinking and Working Like a Scientist, Personal Gains Related to Research Work, Skills, Attitudes and Behaviors as a Researcher, Satisfaction categories).

- Above average grit scores and confidence in success.

- $\sim 75 \%$ of mentors describe above or well above average student performance. 


\section{Rate how much you agree with the following statements.}

My research experience has prepared me for a job.

My research experience has prepared me for graduate school.

My research experience has prepared me for 4-year college.

My resume has been enhanced by my research experience.

My research experience has prepared me for advanced coursework or thesis work.

Doing research introduced me to a new field of study I want to pursue.

Doing research clarified for me which field of study I want to pursue.

Doing research confirmed my interest in my field of study

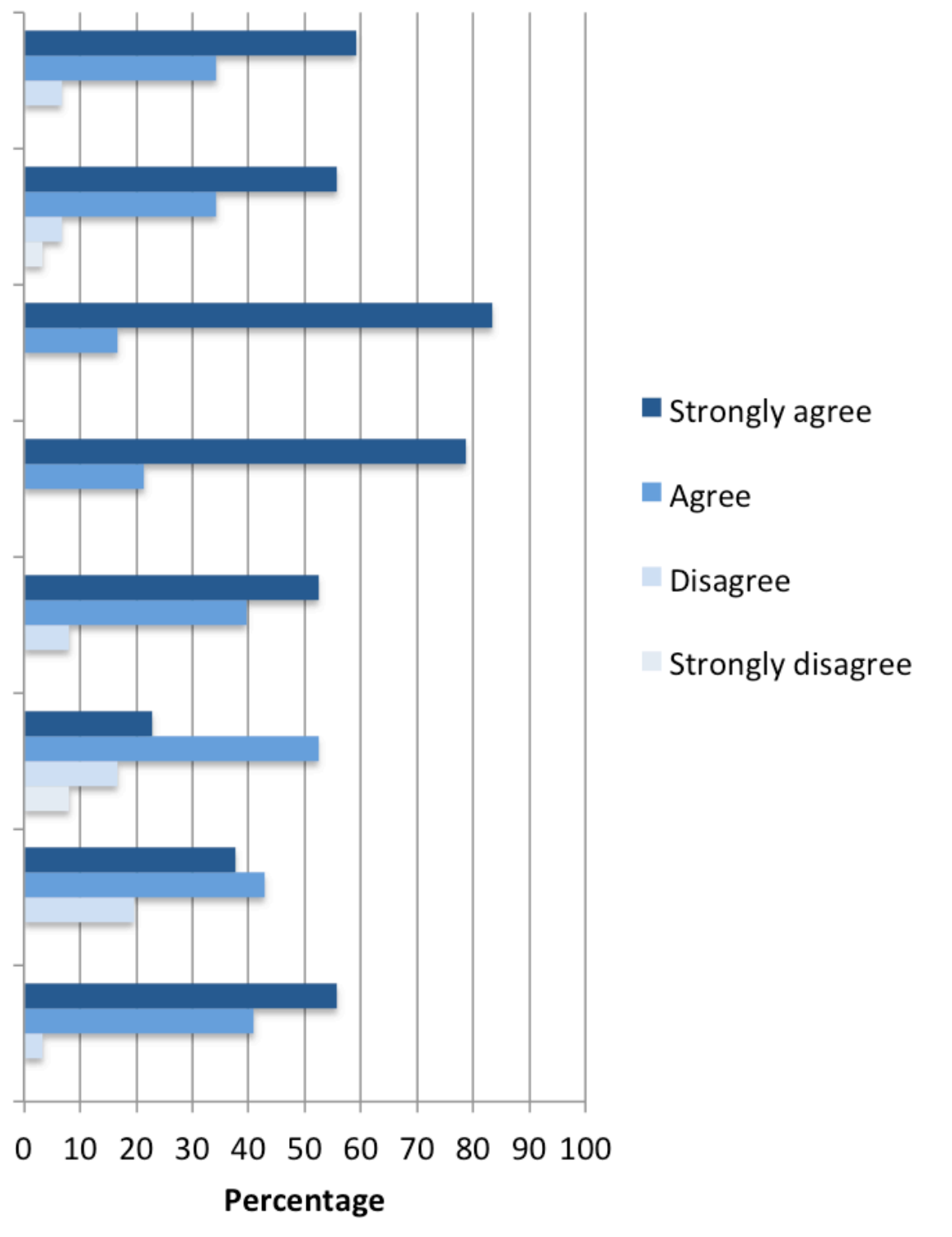




\section{Take Away}

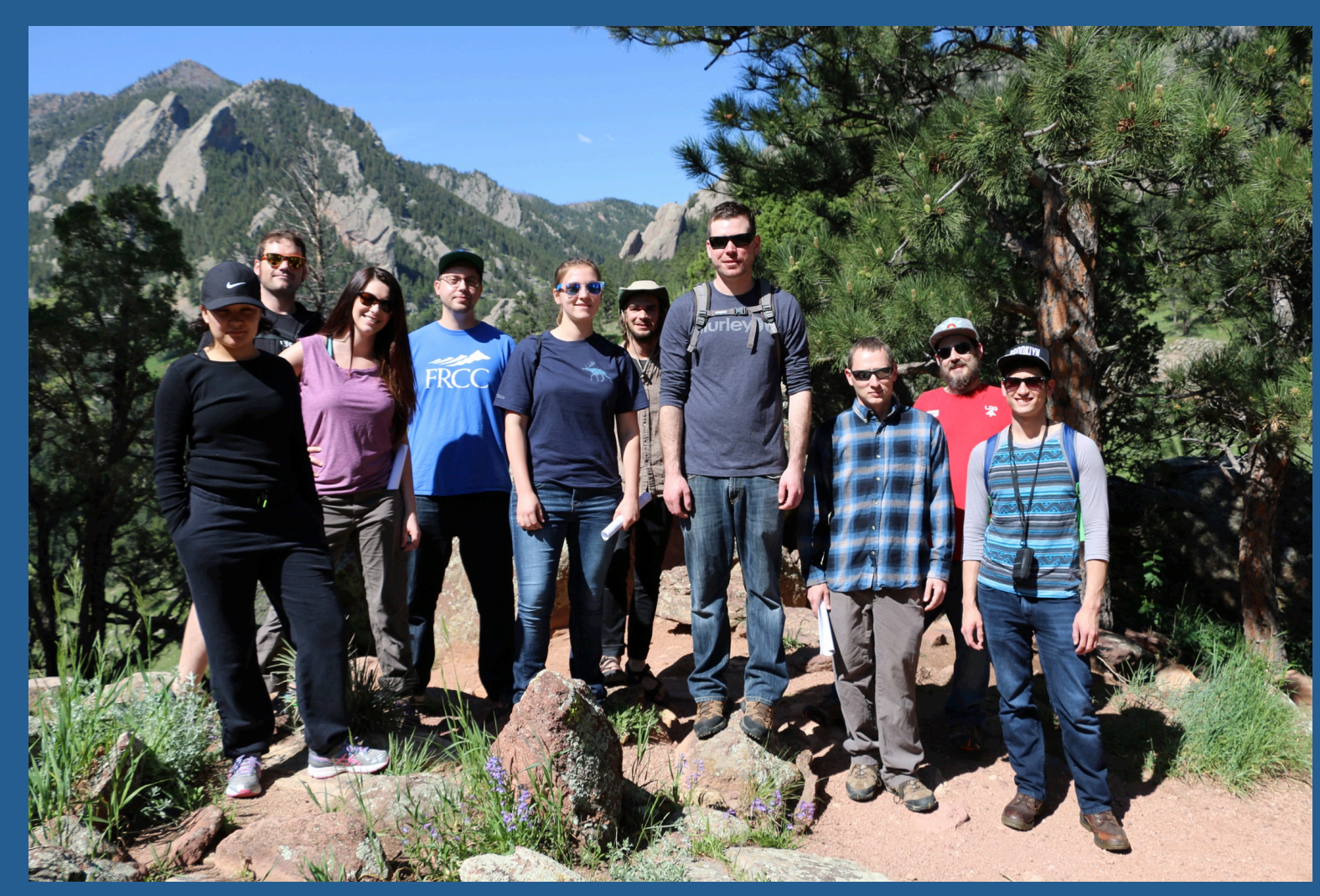

"RECCS has given me such an boost in self esteem, what I am capable of-I never knew because I had never tested myself. I owe you all such a debt of gratitude, I hope one day I can pay it back. Until then, please be well, and know that you changed the life of at least one person, for the better." 


\section{More Information}

Lesley Smith

Lesley.Smith@Colorado.edu

Anne Gold

Anne.U.Gold@Colorado.edu

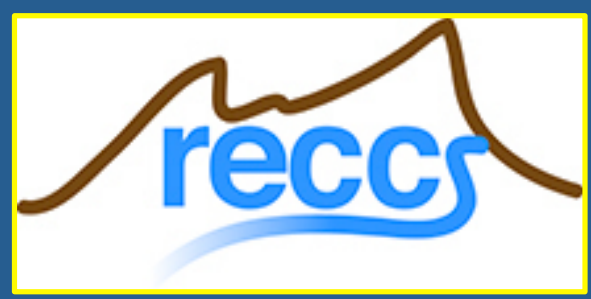

cires.colorado.edu/outreach/reccs

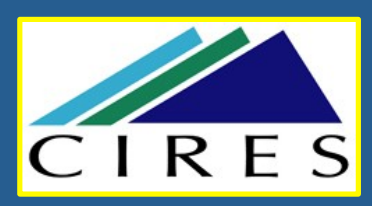

